\title{
Scaffolding Social Presence in MOOCs
}

\author{
DILRUKSHI GAMAGE*, University of Moratuwa
}

Massive Open Online Courses (MOOCs) often lack valuable social presences that provide learners opportunities to interact and to be feel as a real person. This subjective experience of being present with a "real" person and having access to their thoughts and emotions increase the learning experience. Although MOOCs provide forums for such interactions, they are often populated with cognitive types of presence limiting social presence. Meaningful social interactions are challenging in MOOCs due to scale and design limitations. This research presents a conceptual framework of 4 phases: Cluster, Orient, Focus and Network to scaffold better social interaction in MOOCs. The framework introduce a re-design of interactions in MOOC forums space - leverages clustered groups by creating many communities in forums spaces with a community leader facilitating the conversations leading social presence. It also provides a community structure with orientation, focus to work in the community and better networking opportunities. An initial integration of the framework to a real MOOC with 27, 554 students provides preliminary results indicating effective scaffolding of social presence in MOOC communities, yet the framework poses challenges relying the social interactions centered upon community manger. However, the study opens new directions to leverage communities in MOOCs and potential to scale.

CCS Concepts: • Human-centered computing $\rightarrow$ Collaborative and social computing systems and tools.

Additional Key Words and Phrases: MOOCs.Social Presence, Sense of beloning

ACM Reference Format:

Dilrukshi Gamage. 2021. Scaffolding Social Presence in MOOCs. In Asian CHI Symposium 2021 (Asian CHI Symposium 2021), May 8-13, 2021, Yokohama, Japan. ACM, New York, NY, USA, 11 pages. https://doi.org/10.1145/3429360.3468198

\section{INTRODUCTION}

Massive Open Online Courses (MOOCs) often face challenges due to their core design - being open and massive. Openness enables any participant to enroll to any course at anytime and as a consequence, it is likely that at any given time there is a significant number of students enrolled with fluctuating interactions in courses than at any typical closed online course [13]. In MOOCs, student interactions are often limited to the space in the forum, yet researchers have repeatedly claimed forums are either disorganized, unused or lead to chaos with massive discourses [7]. On the other hand, researchers claim - for a better learning experience, social interactions are crucial and MOOCs are notoriously labeled as being lacking in social presence [1]. Social Presence is the sense of having a real person in the community leading to enhanced learning experience. However, limited interactions occur in MOOCs relating to social presence in forums. Social discussions often take place outside of MOOC platforms such as Facebook, Twitter, and other social networking sites due to the lack of features supporting such interactions in MOOCs [24]. Although there are learning analytic evidence highlighting the lack of social presence in MOOCs [15], interventions that scaffold social presence in MOOCs remain significantly limited, specifically interventions utilizing existing resources.

Permission to make digital or hard copies of all or part of this work for personal or classroom use is granted without fee provided that copies are not made or distributed for profit or commercial advantage and that copies bear this notice and the full citation on the first page. Copyrights for components of this work owned by others than ACM must be honored. Abstracting with credit is permitted. To copy otherwise, or republish, to post on servers or to redistribute to lists, requires prior specific permission and/or a fee. Request permissions from permissions@acm.org.

(c) 2021 Association for Computing Machinery.

Manuscript submitted to ACM 
Embedding social presence is ongoing and largely explored in human-computer interaction specially in human-agent interactions [14], AR and VR [10] and socio-technical systems such as online communities [11]. Especially the social engineering for asynchronous digital communities are increasingly important since such technologies are overarching our behaviours such as the ways we work, play, learn, conduct commerce, organize politically, and receive social support. Success of socio-technological platforms for communities are much dependant on its engineered features for recruiting and socializing members, developing members' commitment, eliciting contributions, regulating behavior and coordinating work [16]. Although much research exists for designing digital communities specially for online learning [5], significant attention has not been given to designing effective online communities for newest online learning phenomena - MOOCs. Existing socio-technical platforms for MOOCs are significantly lacking social capital the interactions that bring social presence among members and enhance learning experience. When designing social interactions for learning systems, inspiration can be drawn from successful online learning communities specially when designing social affordances to learning communities [8]. Based on such design features, this paper introduces a 4 step framework to model social presence in MOOC platform designs - Cluster, Orient, Focus, and Network (Fig. 1). Exploring MOOC participants motivations and behavioural implications in existing platforms, it is evidence that MOOC users found it hard to navigate meaningful interactions. Specially since there are massive number of participants in the forum, the congitive interaction culture and MOOCs users expectations of active guidance [19] are challenging for platforms to bring social capital. This framework provides a design mechanism which enables MOOC platforms to articulate social presence by incorporating social features which bring meaningful social interactions yet using the existing forum structure in MOOCs. Clustering divides the forum space into groups while identifying a facilitating leader. The leader is oriented to take the lead in communication and keep engaging with their peers. The leader will then focus on community building and subject discussions while enabling sense of community. This stimulates networking where peers will get to know each other more while learning in a short span of time.

The paper presents findings from a MOOC on the OpenSAP platform with 27, 554 registered participants in which the framework was used to scaffold social presence. The results found evidence of social presence and a potential avenue to scale using a network analytic approach. In follow-up work, active integrating mechanisms for many MOOC platforms are needed where it will provide a concrete understanding of the causal implications of this framework. At the same time, recommends to examine its longitudinal effects on learning outcomes and social presence. Although these are early stage results, this finding brings uniquely practical insights to MOOC designers who hope to induce social presence and scale it effectively.

\section{RELATED WORK}

Social Presence found to be a key factor contributing to higher learning experience in MOOCs. However, behaviour analytic research constantly provides evidence for lack of social presence in existing MOOC designs [15]. As a solution, researchers found peer support in forum [2], effective platform designs, including gamification [1] or off-platform social mechanisms [18] may provide potential for MOOCs to bring social presence. Yet, social presence in MOOCs has been underserved in terms of understanding effective interventions and scaling it and such intervention needs pedagogical changes to MOOCs - sometimes extra infrastructure support since existing MOOC platform designs do not provide affordance for such features. MOOC platforms by its design concentrate on content dissemination and less focus on features provide for participants to engage with each other. Forum remains the only space where participants can interact during the course. By its design, forums in MOOCs are ineffective to engage meaningful interactions and thereby do not lead to social presence in the course. However, HCI and behavioral social science research introduce 


\section{Entire MOOC}

\section{Clustering}

\section{Orienting}

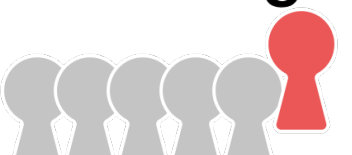

\section{Focusing}

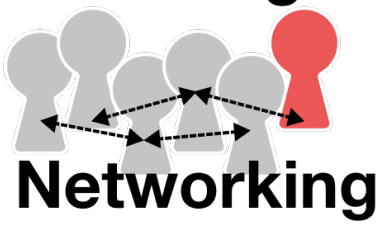

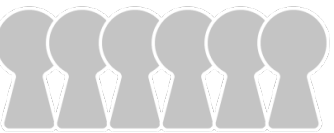
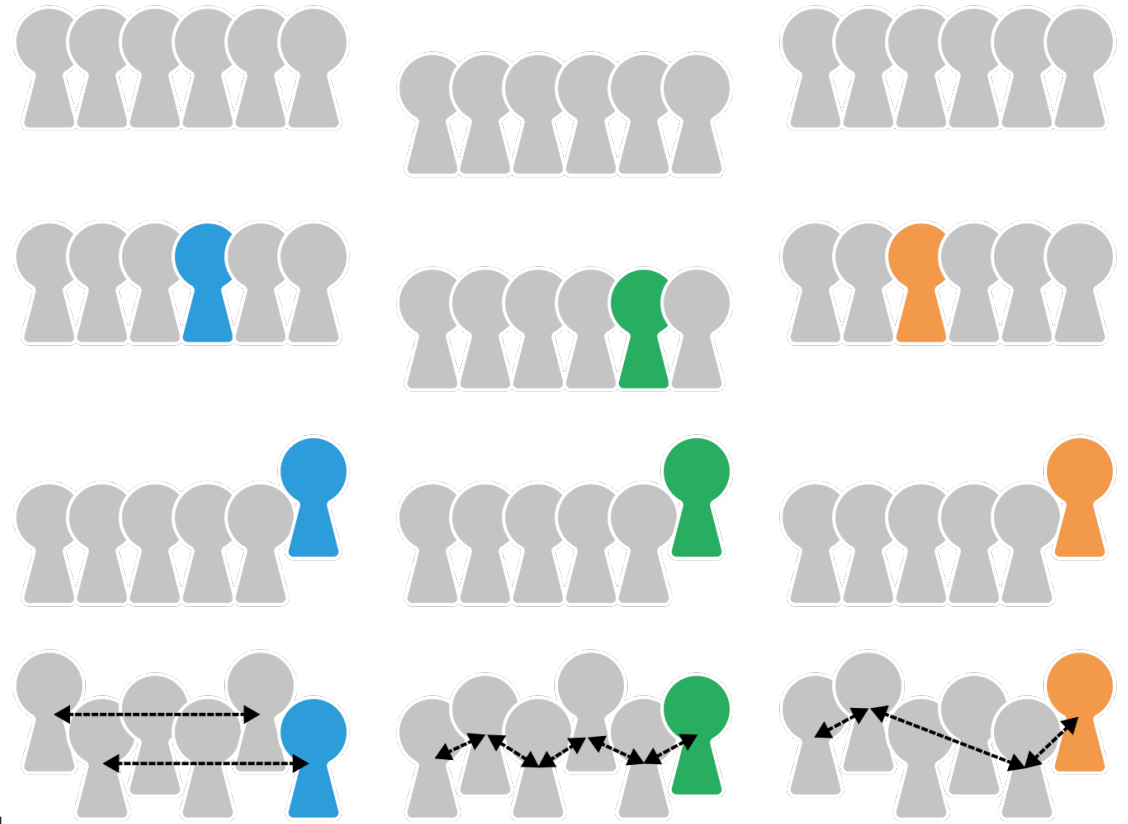

Fig. 1. This reflects the framework where initial MOOC participants will be clustered (not necessary to be equal numbers), identify a facilitating leader (colored), Community leader focusing and directing discussions, regular discussions in closer groups stimulate networking and thereby induce social interactions.

social presence theory related to building communities [17], and empirical research on building communities [12], small groups and organizations, which underlie design decisions leveraging forums such as in open-source software development projects, health support groups, and massively multi-player games [23]. MOOC forums can re-design leveraging under pining theories of digital communities and its design to impend social presence.

Much research aims to understand the nuances of social presence in MOOCs $[9,15]$ but there remain important shortfalls in this line of work [13]. In order to bring social presence to MOOCs, researchers have applied Gamification and off-platform social mechanisms [18].

Currently, forum re-design's and building successful communities within the MOOC platform is very much lacking. To obtain social presence, unlike other methods, this research conceptualize a framework which reflects features of communities in digital spaces. Drawing on the concept of facilitation [6] and borrowing practical guidance for building online communities [11], that bring social presence, this paper present a framework for MOOCs which has 4 phases. 


\section{OVERVIEW OF THE FRAMEWORK}

To bring social presence to MOOCs, the research conceptualized articulating prior theories in community design and online learning experience. Considering the typical flow of a MOOC and its practical conduct, the paper drew insights on the aspects or features to consider. In a MOOC, thousands of students enroll at any given time. Many MOOC platforms provide a forum space to interact. However, due to the wideness and openness of this space, the discussions tend to overwhelm and are difficult to navigate or have meaningful conversations leading to social interaction. Borrowing the concept of connectivist structure (cMOOCs) [4], it is plausible to bring community aspects of social interactions even when the network is loosely coupled and has many participants. According to the principles of community building, social capital can be obtained by structuring the community such that it encourages comments, contributions, welcomes newcomers, and regulates behaviour [11]. This notion can be articulated into a framework to scaffold social presence in MOOC environments. The phases of the framework are detailed below and depicted in Fig. 1.

Clustering is the action which brings participants into a narrow space so they can gain visibility and build meaningful connections. Clustering a MOOC results dividing learners into groups with a small number of participants with forum spaces for each group. At this stage proposes a randomised or interest based grouping, however the grouping mechanism can be optimized in future.

Orienting is bringing the small group clusters into a common understanding. Each cluster will introduce a new community role - community manager and community participants. Community participants will be welcomed by a selected community leader as the main facilitator. Leaders could be selected in a variety of ways such as through prior experience or evaluation. The simplest, and one in this framework used is through selecting volunteers from the main participant pool who also have enough time to contribute in this capacity. During orienting activities, all participants are given community guidelines and provided information how to be engaged and work in the community.

Focusing is when the cluster's community manager or the leader regulating the forum space while encouraging comments and social interaction. Community leaders are given instructions to conduct sessions or close facilitation to bring the community together, coordinating the discussions. This work involves helping to balance academic and social discussion in the group.

Networking is when the clusters closely communicate with each other, reflecting, posting, liking, replying to threads in the forum, and taking part in any online sessions organized in the community. This is mainly the members building social capital by making friends and bonding behaviors. While focusing the engagement, in a small scaled forum space, ongoing conversations strengthen the social network.

\section{METHODS}

The initial conceptual idea of the framework was incorporated to a MOOC - Object-Oriented Programming in Java, which was offered in the OpenSAP MOOC platform from June, 13th 2018 to July, 26th 2018. The research was under ethical clearance to handle the pseudonymous data complying with the General Data Protection and Regulation (GDPR). Unlike most other MOOC platforms, the OpenSAP platform includes a feature, so-called "CollabSpaces", that could use to create separate spaces for communities within the overall course community. These can be configured to be open to anyone in the class or they can be defined as a closed group space that requires an invitation to join as clustered groups. Apart from forum space in the CollabSpace, the courses usually utilize the main forum space as a general forum to discuss course content with participants.Figure 2 provides the interface of the Collabspace in the OpenSap Platform. 


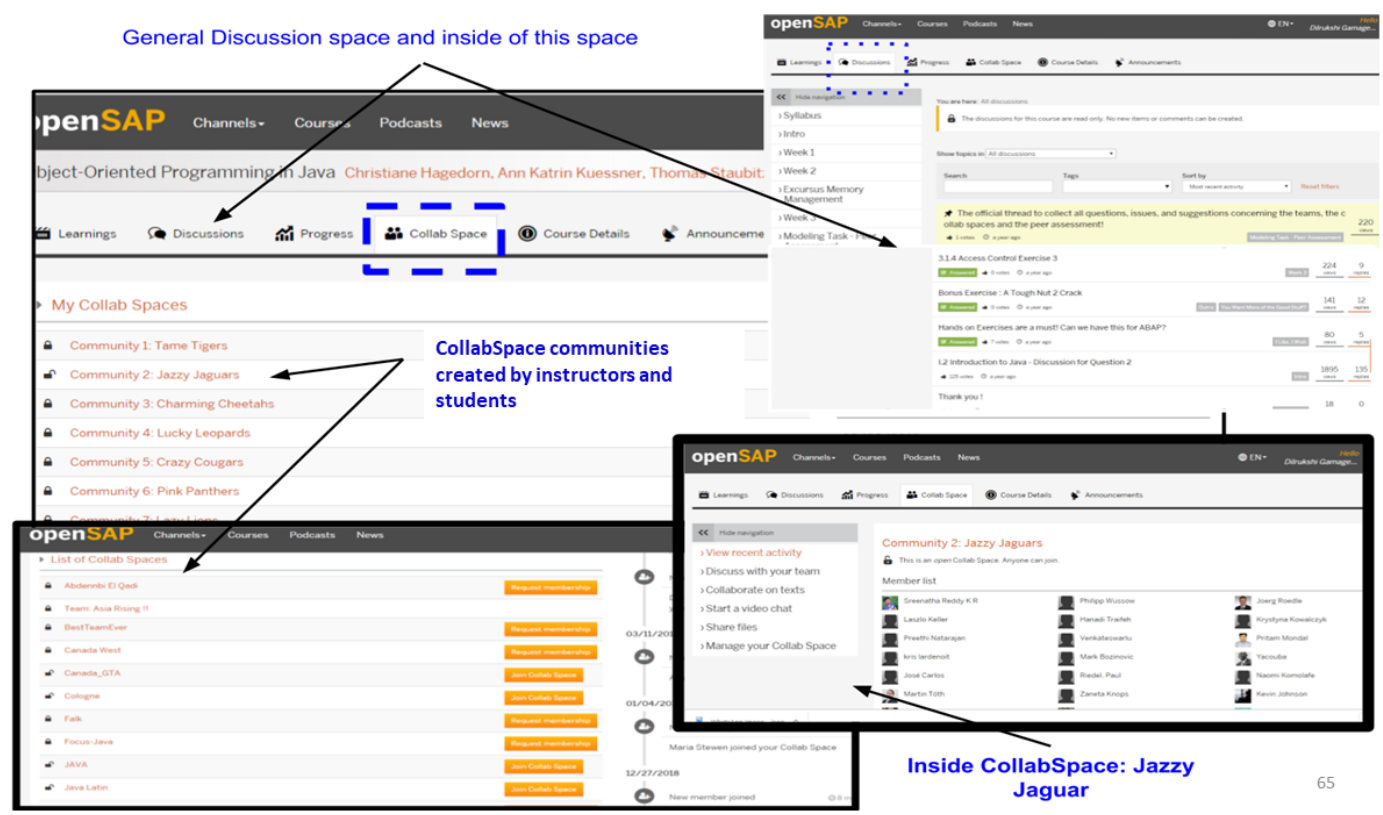

Fig. 2. Collab Space based on the experiment design.

\subsection{Applying the Framework}

To reflect clustering, the space used from the CollabSpace and created designated forum spaces. This creates few small communities than the entire space in the forum for MOOC participants. From the participants who enrolled in the course, community manager roles were invited so they could be assigned to any space created in the CollabSpace. Initially the clustered spaces were assigned names for uniquely identifying the activities occurring and invited any participant to take the leading act in each cluster. Joining this community was optional and there were no additional benefits allocated in terms of course credits. This constructed forum space consisted an artificial stimuli for community building by introducing clear instructions using a community manager role and specific community instructions which reflect orient and focus phases of the framework. The group in this space was constantly encouraged to lead community building activities, conduct online meetings, discuss off-the topic social bonding stories reflecting the Network phase. However, there were students who did not join the space we created as clusters but continued the course as a regular student taking part in course general discussions.

\section{EVALUATIONS AND RESULTS}

The MOOC had 27,554 registered participants, of which only 24\% participants obtained a Record of Achievement by earning at least $50 \%$ of the available points in the exams and exercises. $39 \%$ of the participants obtained a Confirmation of Participation by accessing at least $50 \%$ of the course material.The course lasted 6 weeks and was intense with hands-on programming exercises, quizzes, weekly exams, and a final exam. While incorporating the framework into the MOOC, evaluations focused on understanding behavioural changes and social interactions in the clustered groups 
Table 1. The categories of content populated in the MOOC forum

\begin{tabular}{c|c|l}
\hline Name & ENA code & Description \\
\hline Social Task & S.Task & $\begin{array}{l}\text { Participants' sense-making of their ability to complete } \\
\text { course tasks or understand course content such as } \\
\text { grouping, communications }\end{array}$ \\
\hline Cognitive Task & C.Task & $\begin{array}{l}\text { Non tasks are specially focused on social aspects, i.e. } \\
\text { introductions, norm setting or the purpose of MOOCs }\end{array}$ \\
\hline Cognitive Non Task & C.NTask & $\begin{array}{l}\text { Non tasks were the conversations anything about sub- } \\
\text { ject but not in the form of quizzes and questions such } \\
\text { as sharing new problems in the subject area }\end{array}$ \\
\hline Administrative Task & Admin & $\begin{array}{l}\text { The conversations relating to course administrative } \\
\text { problems such as when is the assignment submissions, } \\
\text { delay requests }\end{array}$ \\
\hline Error Reporting & Error & Error reporting in the site or course , software modules \\
\hline
\end{tabular}

in the framework. This evaluation provides insights into impending social presence and understanding the interaction types in the MOOC.

\subsection{Type of interactions and social presence}

To understand the interactions in the MOOC required to identify the activities occurring in forum space in the MOOC. Analysing forum discourse leveraged Epistemic Network Analysis (ENA) ${ }^{1}$. ENA is a quantitative ethnographic method and generally used to operationalize the building of the knowledge in qualitative data [20]. In this case, the study used ENA to build knowledge structure from forum messages to observe types of interactions. Knowledge constructs on social aspects in MOOCs were categorized based on previous literature where researchers evaluated social presence in MOOCs content $[9,15]$. The forum posts in the MOOC were categorized and coded in the CollabSpaces which created according to the framework. According to previous literature, forum messages are generally categorize based on if the conversation is reflecting a cognitive task, social task, social non-task, or cognitive non-task [15]. In this case, study found new categories of administrative and error reporting conversations as well. ENA takes the categorised codes and their occurrence frequency to build a ENA weighting on these categories as Fig 3. Forum posts were coded according to these categories by 2 coders and their inter-rate reliability Cohenś Kappa (0.79) were with in substantial agreement.

Focusing on the relationship between the codes in the weighted network diagram which highlights the interaction, the study highlights the strong connection in social tasks and non-social tasks in CollabSpace which incorporated with framework. Social tasks are participants conversations relating to forming communication methods, norm settins and non-social tasks are specifically the introductions of friends, personal stories. However, non-social tasks and cognitive tasks also made to second strong association. Cognitive tasks are the conversation about the subject related discussions that arose during assignments or exercises. There was significantly less association between Administrative conversations and Error Reporting in this forum. This resulted highest weight in the social task and non-social task than any other category and in other words, the social presence was the highest among other discussions in the forum using the framework.

\footnotetext{
${ }^{1}$ More detailed explanation of these methods [21] and tutorials on using them [3,22] are available.
} 


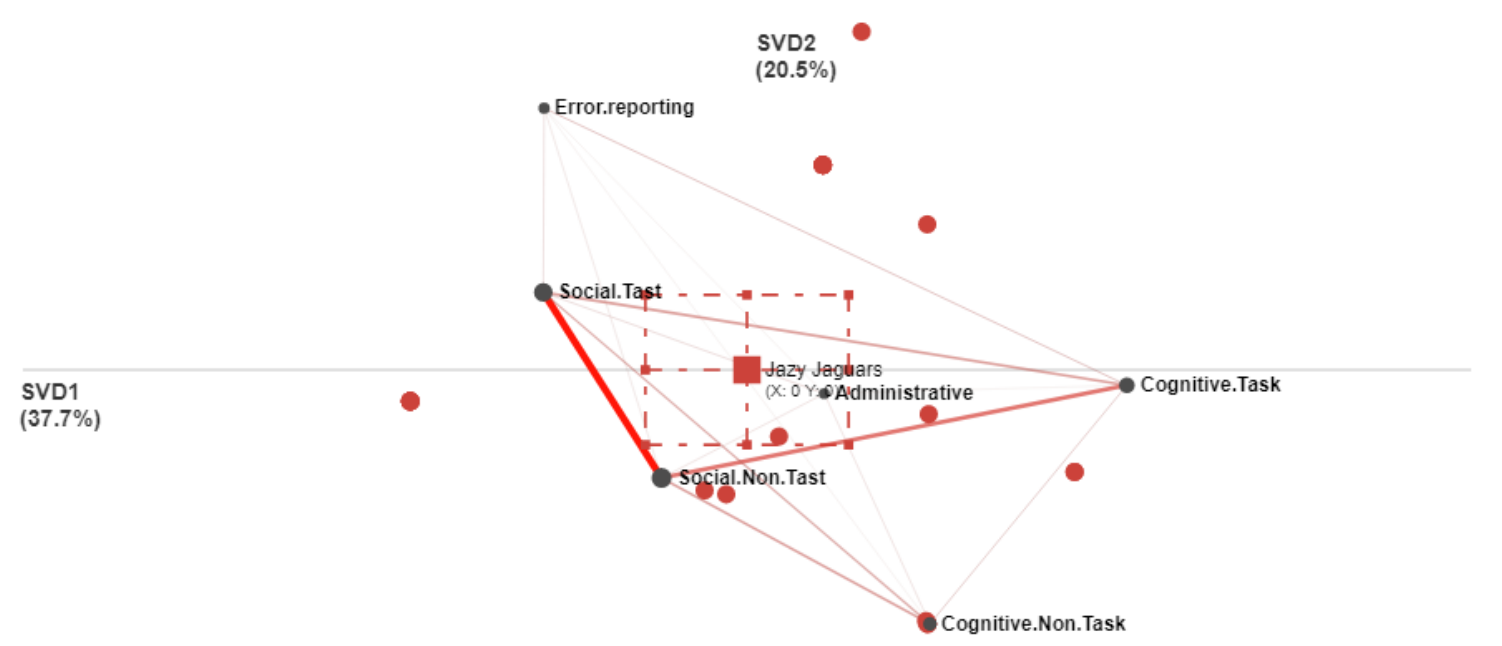

Fig. 3. ENA graph which reflects the type of interactions. Strength of each connection where higher values with stronger connections in thicker edges. There were several strong connections within the social presence indicators, such as the link between Social and non-Social task (2.85) and Social non-Task and Cognitive Task (1.65).

\subsection{Reflected social behaviours from the Framework}

The study reports observations of the MOOC which had the framework integrated to the forum space. During the study - the framework was executed with no changes to the MOOC platform and while utilizing the existing forum space. In this case the CollabSpace feature in OpenSAP platform provided isolated communication clusters. In earlier MOOC platforms, forums were chaotic and caused cognitive burden [25]. Integrating the framework, the study reflect significant behavioral changes in the discussions. These discussions can be identify in two distinct categories-Community manager initiated discussions; Community Participants initiated discussions.

At the beginning of the course, many confusions were observed among participants, mainly due to unfamiliarity of the new community concept. However, after the orienting the participants towards the community which brought clear instructions on how the group will move, the discussions were more directed towards activities in the course and socializing. Following quotes from a participant, reflect an example incident of being confused.

"I am not sure what I have to do here, will there be someone to guide me or should I start continuing the course. Can someone let me know some information in this group."

The following quite can be highlighted to reflect the participants adhering to the community norms and guidance in the orienting phase of the framework.

"Thanks for updating the information, also thank you for your time, I have been thinking about how we can proceed in this group and weekdays anytime between 5-8 PM CET time is feasible for me to have a town-hall meeting"

Community managers had a prominent role in the framework, which needed an effort to coordinate the cluster and lead communications as a facilitator. Clear instructions for the community managers were found to be useful as they are also new to the concept of facilitating and leading community in a MOOC. The following quote from a community manager reflects the experience. 
"Hi all, I have volunteered to lead this group, not sure how I can do it, but I have given some instructions on how to conduct the sessions. As it explains, we can start by greeting each other and getting to know in this thread, I will try to reach you to see how can we fix a time for a meeting if possible, or should we continue our discussions in the forum only? I am ok with either but prefer if we can have at least 2 online meetings. "

Although the framework was integrated to the real MOOC to design a community structure which could lead social presence - while conducting this evaluation, it was challenging to identify and appoint community leaders who are willing to serve as facilitators. Since they play an active role, this study emphasizes the need for a facilitator with an incentive-driven model to encourage good participation.

During the 6 weeks of the course, it was observed in the main discussion forum (not the CollabSpace which had incorporated the framework), several very active students who kept responding to others posts. These participants reflect community behaviours. However, since there were a large number of threaded discussions, their active involvements were faded without leading any networking or socializing with members. CollabSpace communities well utilized these active participation as such types of interactions were well received by the community and lead to rich discussions igniting the sense of community, norms and values in the space.

The observations revealed that this approach uncover community members who can play a roll as facilitators and reflects the potential of the scaling the model of clustering. Since the framework does not encourage cohesiveness as a closed bond team work, but rather the elasticity of the participation, reflecting the community, it is possible that students interchange between communities. The concept can be easily implemented in other platform designs to optimize social presence at scale.

\section{DISCUSSION AND IMPLICATIONS}

This paper provides an initial conceptualised framework for MOOCs which could structure the missing social components in learning. Although learning analytic researchers have repeatedly provided evidence of the lack of social presence in MOOCs [15], interventions have not been successfully implemented. It needs careful design considering the learning experience borrowing from the field of human-computer interaction. This study leverages social presence theories and conceptualizes the actionable steps can be taken in a learning at scale situation. Implications of such actionable steps in a framework will provide insights for platform designers to implement interaction designs in MOOC platforms which enable the features required for a successful learning community in MOOC.

\subsection{Rapid Community space for social learning}

The introduced framework supports to create rapid communities to for peers to learn together as a loosely cohesive group. It also provides a semi-structure by introducing a community manager, a guide to the community manager, and members and supporting tools in the platform to facilitate community practice. In a typical context, building communities takes time and effort to find, groom the leaders with the time and members grow or shrink based on community practice [11]. CollabSpace provides inbuilt facilities to scale social interactions by providing visible identities in their profiles and active facilitation by the community manager. In other words, this mimics the learning circles in peer2peer university into fully online [? ]. However, the framework enables scaling and sustainable logistics than in physical conditions, providing more accessible solutions for wider MOOC participants. Although this is intended for MOOC courses, this setup can be wide applicable to any online course where a team of the course engage while they learn and lead to an effective social learning experience. 


\subsection{Growing and sustaining learner community}

Success of the framework and rapid communities are highly depended on the activities of members. This study witness the difference based on the number of members and community manager. Although proving the tools in the virtual space, the framework model is highly dependent on community managers effectiveness. Members often tend to interact with personal address, name calling and cheer leading the discussions, which is a prudent action item for community managers. However, at this stage this role is completely voluntary. Similar to a model which recruits interest alumni [? ] to act as facilitators in MOOCs or a model similar to hiring peers [? ] provide insights to the framework to improve the sustainable model for community manager engagements. We learned that some members less interact and some highly interact in comments and likes in threaded discussions while taking part in online meetings. To maintain higher average engagement levels, it can draw best practices from communities and articulate such with the designed system. Features such as gamifications to the community [? ] have proven to increase volunteer contributions, which can be incorporated it in the future.

\section{3 limitations and future work}

Without no doubt, this framework has the potential for improvement. The first priority of this conceptualized framework was to capture social presence in the designed communities in MOOCs. The process that incorporate to create a space and enable cluster many communities, orient with community manager and member guidance, focus on the discussions relating to MOOC and expand opportunities to network - may not be the only solution to impend social presence and incorporate communities of practice. It is imperative to bring more solutions to MOOCs which enable social learning and community engagement since MOOCs are fading by driving the concentration of content but limiting the social interactions. At the same time, this study conducted to evaluate the framework had limited subjects and only one MOOC was used to evaluate the idea. The selection of a computer science course may impact the results compared to if the subject were related to more discussion-based subjects such as liberal art, logic, and philosophy. In the future, more MOOC platforms need to integrate the framework and explore the social behavioral aspects and enhanced learning experience in the learning platform.

\section{CONCLUSIONS}

This study introduced a framework to increase social presence in large scale online learning - MOOCs. HumanComputer Interaction researchers found ground evidence of Social presence as a motivational factor for increase learning experience in MOOC participation, specially how interactions in the forums of large scale online learning can be re-design to increase quality of learner experience. This research introduces a four phase framework - Cluster, Orient, Focus and Network which provides a specific articulation - a mechanisms to scaffold social presence using the forum space in MOOCs. The initial integration of the framework to a real MOOC as a field evaluation showed a significant level of social presence among the categories of social interactions. The framework centered around the community manager and community participants, and designating facilitators as community managers and their role being critical and central to this framework posed a challenge. Based on the observations and behavioural analysis, the study propose to leverage on incentive mechanisms to resolve this. The analysis of discourse of the participants indicated that there were highly active participants who could have taken Community manager role and motivate other participants to actively engaged in the community during the course. Large scale studies across MOOC platforms and 
other subjects where social presence may play an influential role are important next steps to understanding the impact of this framework.

\section{ACKNOWLEDGMENTS}

This work is grateful for the support provided by Dr.Thomas Staubitz HPI Germany and Dr.Mark Whiting at University of Pennsylvania and Prof. Michael Bernstein at Stanford University for helping to shape the the initial conceptual ideas and for the valuable feedback provided.

\section{REFERENCES}

[1] Alessandra Antonaci, Roland Klemke, Johan Lataster, Karel Kreijns, and Marcus Specht. 2019. Gamification of MOOCs adopting social presence and sense of community to increase user's engagement: an experimental study. In European Conference on Technology Enhanced Learning. Springer, $172-186$.

[2] Kwamena Appiah-Kubi and Duncan Rowland. 2016. PEER support in MOOCs: The role of social presence. In proceedings of the third (2016) ACM conference on learning@ scale.237-240.

[3] G Arastoopour, Z Swiecki, NC Chesler, and DW Shaffer. 2015. Epistemic network analysis as a tool for engineering design assessment. American Society for Engineering Education. Seattle, WA (2015).

[4] Ángel Fidalgo Blanco, Francisco J García-Peñalvo, and Marisa Sein-Echaluce. 2013. A methodology proposal for developing adaptive cMOOC. In Proceedings of the First International Conference on Technological Ecosystem for Enhancing Multiculturality. ACM, 553-558.

[5] Chris Brook and Ron Oliver. 2003. Designing for online learning communities. In EdMedia+ Innovate Learning. Association for the Advancement of Computing in Education (AACE), 1494-1500.

[6] Pin-Ju Chen and Yang-Hsueh Chen. 2014. Facilitating MOOCs learning through weekly meet-up: a case study in Taiwan. In Proceedings of the first ACM conference on Learning@ scale conference. ACM, 183-184.

[7] Derrick Coetzee, Armando Fox, Marti A Hearst, and Björn Hartmann. 2014. Should your MOOC forum use a reputation system?. In Proceedings of the 17th ACM conference on Computer supported cooperative work \& social computing. ACM, 1176-1187.

[8] Ben Hanrahan, Sameer Ahuja, Manuel Perez-Quinones, and Andrea Kavanaugh. 2011. Evaluating software for communities using social affordances. In CHI'11 Extended Abstracts on Human Factors in Computing Systems. 1621-1626.

[9] Srećko Joksimović, Nia Dowell, Oleksandra Skrypnyk, Vitomir Kovanović, Dragan Gašević, Shane Dawson, and Arthur C Graesser. 2015. How do you connect?: analysis of social capital accumulation in connectivist MOOCs. In Proceedings of the Fifth International Conference on Learning Analytics And Knowledge. ACM, 64-68.

[10] Kangsoo Kim. 2018. Improving Social Presence with a Virtual Human via Multimodal Physical-Virtual Interactivity in AR. In Extended Abstracts of the 2018 CHI Conference on Human Factors in Computing Systems. 1-6.

[11] Robert E Kraut and Paul Resnick. 2012. Building successful online communities: Evidence-based social design. Mit Press.

[12] Kathy Ning Shen and Mohamed Khalifa. 2008. Exploring multidimensional conceptualization of social presence in the context of online communities. Intl. Journal of human-computer interaction 24, 7 (2008), 722-748.

[13] Poquet Oleksandra and Dawson Shane. 2016. Untangling MOOC learner networks. In Proceedings of the Sixth International Conference on Learning Analytics \& Knowledge. ACM, 208-212.

[14] Andre Pereira, Rui Prada, and Ana Paiva. 2014. Improving social presence in human-agent interaction. In Proceedings of the SIGCHI Conference on Human Factors in Computing Systems. 1449-1458.

[15] Oleksandra Poquet, Vitomir Kovanović, Pieter de Vries, Thieme Hennis, Srećko Joksimović, Dragan Gašević, and Shane Dawson. 2018. Social presence in massive open online courses. International Review of Research in Open and Distributed Learning 19, 3 (2018).

[16] Yuqing Ren, Robert Kraut, and Sara Kiesler. 2007. Applying common identity and bond theory to design of online communities. Organization studies 28, 3 (2007), 377-408.

[17] Ronald E Rice. 1993. Media appropriateness: Using social presence theory to compare traditional and new organizational media. Human communication research 19, 4 (1993), 451-484.

[18] Leon Rothkrantz. 2015. How social media facilitate learning communities and peer groups around MOOCS. International fournal of Human Capital and Information Technology Professionals (IfHCITP) 6, 1 (2015), 1-13.

[19] Tatiana Semenova. 2020. The role of learners' motivation in MOOC completion. Open Learning: The fournal of Open, Distance and e-Learning (2020), $1-15$.

[20] David Williamson Shaffer. 2017. Quantitative ethnography. Lulu. com.

[21] David Williamson Shaffer, Wesley Collier, and AR Ruis. 2016. A tutorial on epistemic network analysis: Analyzing the structure of connections in cognitive, social, and interaction data. fournal of Learning Analytics 3, 3 (2016), 9-45.

[22] Sarah Sullivan, Charles Warner-Hillard, Brendan Eagan, Ryan J Thompson, AR Ruis, Krista Haines, Carla M Pugh, David Williamson Shaffer, and Hee Soo Jung. 2018. Using epistemic network analysis to identify targets for educational interventions in trauma team communication. Surgery 163 , 
4 (2018), 938-943.

[23] Fan-Chen Tseng, Han-Chung Huang, and Ching-I Teng. 2015. How do online game communities retain gamers? Social presence and social capital perspectives. fournal of Computer-Mediated Communication 20, 6 (2015), 601-614.

[24] George Veletsianos, Amy Collier, and Emily Schneider. 2015. Digging deeper into learners' experiences in MOOC s: Participation in social networks outside of MOOC s, notetaking and contexts surrounding content consumption. British fournal of Educational Technology 46, 3 (2015), 570-587.

[25] Miaomiao Wen, Diyi Yang, and Carolyn Rose. 2014. Sentiment Analysis in MOOC Discussion Forums: What does it tell us?. In Educational data mining 2014. Citeseer. 